\title{
The Treatment In Morning versus Evening (TIME) study: analysis of recruitment, follow-up and retention rates post- recruitment
}

\author{
David A. Rorie ${ }^{1,2^{*}}$, Robert W. V. Flynn ${ }^{1}$, Isla S. Mackenzie ${ }^{1}$, Thomas M. MacDonald ${ }^{1}$ and Amy Rogers ${ }^{1}$
}

\begin{abstract}
Background: The use of information technology (IT) is now the preferred method of capturing and storing clinical research data. The Treatment In Morning versus Evening (TIME) study predominantly uses electronic data capture and IT to compare morning dosing of hypertensive medication against evening dosing. Registration, consent, participant demographics and follow-up data are all captured via the study website. The aim of this article is to assess the success of the TIME methodology compared with similar studies.
\end{abstract}

Methods: To assess the TIME study, published literature on similar clinical trials was reviewed and compared against TIME recruitment, follow-up and email interaction data.

Results: The TIME website registered 31,695 individuals, 21,116 of whom were randomised. Recruitment cost per randomised participant varied by strategy: $£ 17.40$ by GP practice, $£ 3.08$ by UK Biobank and $£ 58.82$ for GoShare. Twelve-month follow-up retention rates were $96 \%$. A total of 1089 participants have withdrawn from their assigned time of dosing, $2 \%$ of whom have declined follow-up by record linkage or further contact. When the TIME data are compared with similar study data, study recruitment is very successful. However, TIME suffers difficulties with participant follow-up and withdrawal rates similar to those of conventional studies.

Conclusions: The TIME study has been successful in recruitment. Follow-up, retention rates and withdrawal rates are all acceptable, but ongoing work is required to ensure participants remain engaged with the study. Various recruitment strategies are necessary, and all viable options should be encouraged to maintain participant engagement throughout the life of studies using IT.

\section{Background}

Randomised controlled trials are the most reliable method of evaluating the efficacy or safety of a drug therapy [1]. Research and clinical studies have evolved to the use of the newest technologies and methodologies, with electronic data capture (EDC) now being commonplace and preferable [2-5]. In parallel, email use has quickly developed to replace face-to-face consultations [6-8] and study screening visits [9]. Email has also been shown to be an effective intervention technique for

\footnotetext{
* Correspondence: drorie@dundee.ac.uk

${ }^{1}$ Division of Molecular and Clinical Medicine, University of Dundee, Ninewells Hospital and Medical School, Dundee DD1 9SY, UK

${ }^{2}$ Medicines Monitoring Unit, University of Dundee, Ninewells Hospital and

Medical School, Dundee DD1 9SY, UK
}

changing participant behaviour [10-13] and an effective method of recruitment to clinical trials [14]. Further research is needed to understand how studies that use information technology (IT) compare with similar conventional studies in terms of efficiency and validity.

The Treatment In Morning versus Evening (TIME) study [15] is a British Heart Foundation (BHF)-funded clinical study comparing morning dosing of usual hypertensive medication with evening dosing to determine if one is better than the other in terms of cardiovascular outcomes. The study is conducted predominantly using an online website and is an example of a study where IT and EDC are used to maximise study proficiency and minimise costs. 
The TIME study uses a novel online methodology and IT to recruit participants, obtain consent, collect baseline data and follow participants. Interested individuals self-enrol on the study website (www.timestudy.co.uk) with a password of their choice and their email address. Those who choose to take part then consent and enter demographic data via the secure website, are followed at routine intervals via automated emails, and are asked to enter patient-reported outcomes (PROs) online. The study has surpassed recruitment expectations, with over 21,000 participants randomised over a 2-year period. However, it is unclear how successful the study methodology will be compared with conventional data collection methods. The purpose of this research paper is to evaluate the TIME study and its online methodology in each of the following areas:

- Recruitment

- Follow-up, retention and adherence

- Email and telephone interactions

\section{Methods}

The TIME study recruited participants using various recruitment strategies: radio advertising, posters in general practitioners' (GPs') practices, newspapers, direct email and letters from GPs, hospital clinics, pharmacies, and research study databases of individuals who had agreed to be contacted about research. Interested individuals were invited to register on the study website, to submit how they heard about the study, indicate their consent to participate, and enter the demographic information and medical history data necessary for the study (Fig. 1). Participants were also asked to provide contact information for a nominated surrogate who could be contacted in the event that email contact with the participant was lost. A copy of the participant's consent form was sent to the participant along with an email confirming their allocated time to take their hypertensive medication (morning or evening). Follow-up emails are sent on a 3-monthly basis to assess adherence to allocated dosing time and to identify any potentially related adverse events that the participant has experienced. Owing to the potentially poor quality of data from PROs [16], record linkage to nationally held hospitalisation and mortality data is used to identify potential study endpoint events. Participants are able to contact study staff at any point during the study using a Freephone number and by email or post. Data on study contact by participants were collected during active recruitment by GP practices and after recruitment by GP practices had ceased, in order to assess resource use during the recruitment phase of the study.

To evaluate the study methodology, a review of published literature was conducted. The PubMed library was searched using general search terms with the aim of

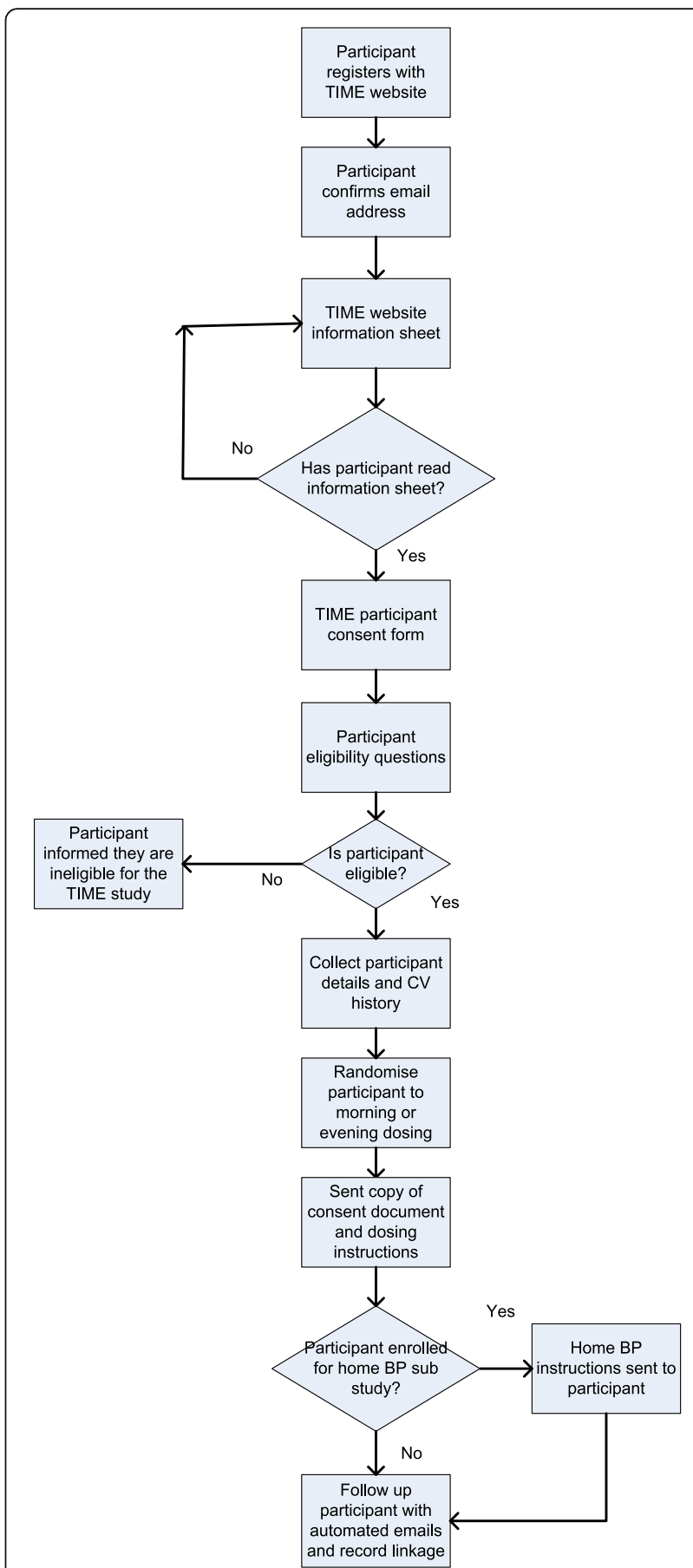

Fig. 1 Flow diagram for Treatment In Morning versus Evening (TIME) study website. BP Blood pressure, CV Cardiovascular

identifying average cost per randomised study participant, retention rates, rates of loss to follow-up, withdrawal rates, and email use and interactions in clinical trials. The remainder of this article compares the TIME study data with the study data identified from the literature review, highlighting the studies' successes and limitations as well as what should be investigated in further research. 


\section{Results}

The TIME study began as a feasibility pilot [17] in December 2011. On the basis of data derived from the pilot study, the BHF funded TIME to recruit 10,269 participants into a formal study. To accommodate the decreasing cardiovascular event rate observed in other studies [18], the recruitment target was increased to at least 21,000 . It was believed that doubling the recruitment target would ensure achieving the necessary number of events needed to power the study within a reasonable time frame. This increase was made possible by the success of the various recruitment strategies used.

\section{Recruitment}

Active recruitment into the study closed in August 2016, and the TIME study website closed to new registrations at the end of March 2017. The majority of participants were randomised between March 2015 and July 2016 $(20,132$ [95\%]), largely due to the work of clinical research networks and GP practices in sending letters to patients with hypertension. During this period, the most successful month was April 2016, in which 1989 participants were randomised. The least successful month, March 2015, saw 421 participants randomised. On average, TIME randomised 1184 participants per month over this period. Overall, between the pilot start date, December 2011, and the registration closure date, August 2017, 31,695 individuals registered with the TIME study website. Of these, $23,617(72 \%)$ met the eligibility criteria ( $\geq 18$ years old, not currently in a study, having a valid email address, and receiving one or more antihypertensive drugs) and went on to complete the online consent process. A total of 2502 (8\%) individuals consented, and were eligible, but did not complete the enrolment process and go on to be randomised into the study. A total of $21,116(67 \%)$ individuals went on to be randomised into the study and were allocated a time of dosing for their antihypertensive medication (Fig. 2).

The TIME study was advertised to potential participants using various strategies. One month after the pilot study began recruitment, data were captured on how registered visitors to the website had come to hear about the study. Each recruitment strategy varied in cost and effectiveness; this is detailed in Table 1 . In an effort to expedite recruitment, UK Biobank [19] and GoShare [20] were recruited to assist in contacting individuals and making them aware of the study. UK Biobank and GoShare are both health registries whose aim is to improve the prevention, diagnosis and treatment of a wide range of serious and life-threatening illnesses. These databases contain details on individuals who have voluntarily registered, given detailed information about themselves and agreed to have their health status tracked. Over many years these will build into powerful resources to help scientists discover why some people develop particular diseases and others do not. UK Biobank sent 79,628 emails to participants with hypertension in their database at a total cost of $£ 10,000$, or $£ 3.08$ per randomised participant (recruitment rate of $4.1 \%$ ). GoShare sent 12,931 emails to patients with hypertension at a cost of $£ 2000$, or $£ 58.82$ per randomised participant. The largest number of TIME participants were recruited from GP practices that had sent individual letters inviting patients to register on the study website. GP practices sent 594,289 letters to patients known to be on at least one antihypertensive medication, using the Docmail [21] mailing service, at a cost of $£ 277,140$, or $£ 17.40$ per randomised participant (recruitment rate of $2.7 \%$ ). Posters displayed in primary and secondary care settings advertising the study were not as successful [22], initially costing $£ 315$ per randomised

\section{Randomised}

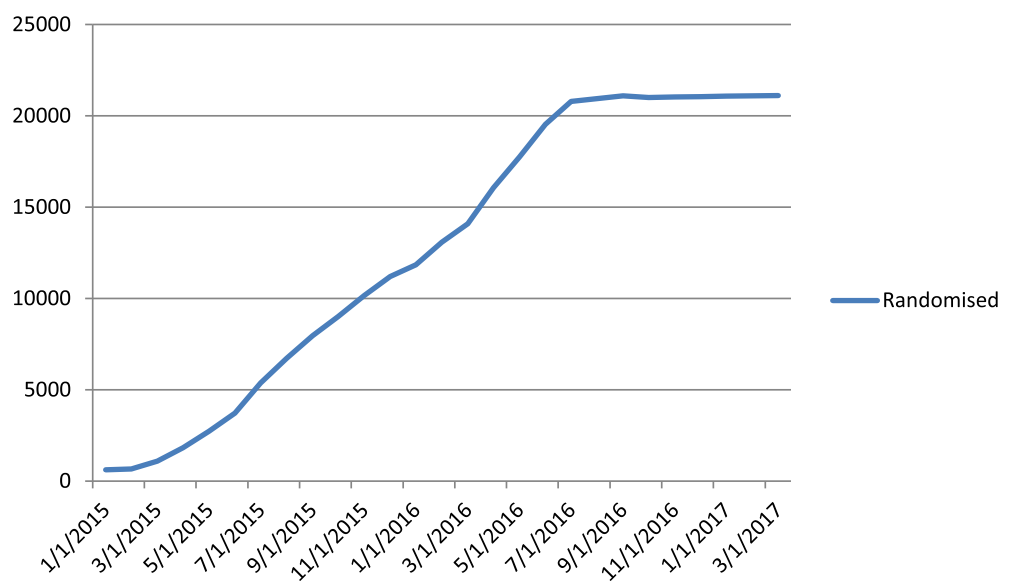

Fig. 2 Treatment In Morning versus Evening (TIME) study recruitment of randomised participants from 2015 to $2017(n=21,116)$ 
Table 1 Treatment In Morning versus Evening (TIME) study registration statistics: how participants heard about the study and cost, if known

\begin{tabular}{llll}
\hline Recruitment method & Registrations $(n=30,775)(\%)$ & Randomised $(n=20,914)(\%)$ & Cost per randomised participant (if known) \\
\hline Radio & $70(<1)$ & $62(<1)$ & \\
Friends/relatives & $102(<1)$ & $65(<1)$ & $£ 17.40$ \\
Web search & $96(<1)$ & $53(<1)$ & $£ 78.00$ \\
From GP & $22,675(74)$ & $15,932(76)$ & \\
MEMO website & $104(<1)$ & $161(<1)$ & \\
Practice poster & $261(<1)$ & $6(<1)$ & \\
TIME video (YouTube) & $14(<1)$ & $3(<1)$ & \\
Medscape & $8(<1)$ & $249(1)$ & $16(<1)$ \\
BHF Heart Matters & $332(1)$ & $8(<1)$ & $£ 3.08$ \\
Pharmacy & $27(<1)$ & $615(3)$ & \\
Newspaper & $17(<1)$ & $3242(16)$ \\
Hospital or clinic & $939(3)$ & $34(<1)$ & $424(2)$ \\
Biobank & $5358(17)$ & & \\
GoShare & $55(<1)$ & $717(2)$ & \\
Other & &
\end{tabular}

BHF British Heart Foundation, GP General practitioner

participant. This cost has been reduced to $£ 78$ per randomised participant as a result of the work of the research networks engaging with practices and distributing additional posters during the recruitment period. Other recruitment strategies used were either free or conducted and funded by supporting bodies, including the BHF, the British and Irish Hypertension Society, the National Institute for Health Research (NIHR) Clinical Research Network, and the Scottish Primary Care Research Network.

\section{Recruitment review}

The literature identified suggests that researchers should use various methods to meet recruitment targets [23, 24]. Over the past decade participant recruitment in clinical trials has not increased, with only $3 \%$ of eligible participants agreeing to take part in clinical research [25]. The response rate to TIME study invitations was similar (4.1\% for Biobank and 2.7\% for GP letters), but the low-cost recruitment strategies implemented allowed for researchers to double the study population to 21,000 from the initial 10,269, despite research governance delays [26]. Involvement of the NIHR Clinical Research Network and the Scottish Primary Care Research Network was invaluable in achieving this.

Trial recruitment costs are reported to vary greatly between studies [27, 28]. Online recruitment has consistently been successful at recruiting more participants in terms of raw numbers and recruiting participants from hard-to-reach populations, and it is more cost-effective than offline methods [29]. However, the technology does bring new challenges. It has been suggested that online recruitment could hinder the identification of well-defined populations and trials reporting PROs have been met with some scepticism about reliability [30]. The TIME study researchers aimed to mitigate this by using PROs to augment a primary study endpoint determined by record linkage to hospitalisations and deaths. To ensure that online studies are generalisable to the target population, it will be important to assess whether participants recruited online are representative of the target population.

Direct contact with study staff is still preferable for some participants [31], and barriers other than household internet access still exist that need to be considered during the recruitment process [32]. During the TIME study recruitment, 8074 people registered but failed to consent or were ineligible, and 2502 individuals failed to complete the enrolment process. The reasons for this remain unknown, and owing to ethical restrictions we are unable to collect these data. Participants clearly responded positively to letters from their own GPs' practices; this appears consistent with similar studies in the United Kingdom using equivalent recruitment strategies [33].

\section{Follow-up, retention and adherence}

PROs are elicited in the TIME study by follow-up emails sent to the participants on a 3-monthly basis. Participants are asked to click on a link within each follow-up email, which automatically takes them to the study website, where they are asked to submit their data. As of March 2017, there were 96,418 completed follow-ups logged on the database. There were a mean of four follow-ups per randomised participant (median of five). 
The most follow-ups reported by a single participant were 25 . Approximately $96 \%(19,882)$ of all live study participants (not withdrawn or deceased) had follow-up information available from within the last 12 months, and efforts are ongoing to contact those participants for whom follow-up data are overdue.

If participants fail to respond to their first follow-up email, they are sent a reminder follow-up email after 14 days. If, after a further 14 days, they fail to respond to the second follow-up email, then an email is sent to the participant's surrogate whom they nominated during enrolment, asking after the well-being of the participant and for any reasons the participant may not have replied to study emails. A total of 6158 surrogate emails had been sent between March 2012 and March 2017, resulting in 669 (11\%) responses from participant surrogates.

In an effort to capture whether participants had read and understood their allocated time of dosing email, a link was added to the allocation email. This link asked the participant to click it if they had understood and acknowledged the time they had been requested to take their hypertensive medication. This link was incorporated into the allocation email in October 2015, and $11,483(54 \%)$ of randomised participants received the email link. Of these, 7949 (69\%) participants clicked the link indicating they had received and understood their allocation email. A total of 3471 (16\%) participants have indicated that they have not adhered to their allocated time of dosing during at least one 3-month follow-up period in the study. A total of 1089 people have formally withdrawn from email follow-up since the pilot began in 2011 (11,070,614 patient-days), but only $388(2 \%)$ indicated refusal to be followed by record linkage.

\section{Follow-up, retention and adherence review}

Whilst there are studies conducted on improving participant adherence [34-37], adherence to allocated treatment during clinical studies is under-reported in the literature. Drop-out rates and loss to follow-up are more widely documented. Reported drop-out rates for studies vary greatly, from $0 \%$ to $45.5 \%$ [38, 39]. Reasons for withdrawal are occasionally reported under typically generic categories, with 'patient decision' and 'adverse event' being the most frequently reported [40]. A suggested general 'rule of thumb' is that $<5 \%$ drop-out leads to little bias, whereas $>20 \%$ poses a serious threat to validity [41]. Currently $5 \%$ of participants have withdrawn from TIME. Participants who wish to withdraw from studies owing to being allocated to their unfavoured treatment arm is a concern and indicates a disappointing lack of understanding of the clinical research process, despite extensive pre-consent written information. Such disappointment is known to be common in clinical studies where participants had hoped to be allocated to an active treatment group. Consent information is of high importance because those who are very disappointed often claim they did not receive understandable information [42]. In addressing specific patient concerns, attention to a patient's emotional status and expectations of trial participation have been found to be associated with better adherence [43]. Researchers in a study of cardiovascular events in diabetes (ASCEND) [33] implemented a run-in period before participants were randomised to ascertain protocol compliance. This process helped alleviate the drop-out rate by identifying non-compliant participants early in the study process and before randomisation took place. There are drawbacks with such a run-in period: increase in study costs, prolongation of the study and underestimation of adverse reactions. However, given the detrimental impact of study withdrawals, a run-in period, which does not exclude participants for reasons other than treatment arm non-compliance, may be worth further consideration for clinical studies.

Whilst online recruitment is effective, remote followup strategies do not reduce the loss to follow-up [44]. There are many reasons for loss to follow-up: Participants may move, deaths may be unreported, or the participant may decide to no longer take part without informing study staff. In some instances the participant may simply no longer comply with the study protocol and not explicitly withdraw consent. There continues to be confusion regarding when a participant is formally withdrawn [45], but if the withdrawal of consent is misinterpreted by the study investigator, valuable data may be lost. The most common reasons cited for continuing participation in research are $24-\mathrm{h}$ access to nurses, better treatment of care and a desire to help others $[46,47]$. Retention in a clinical trial appears to depend on expert medical care, supportive staff-participant relationships, involvement with clinically and scientifically meaningful research, and management of participant expectations [48]. The extent to which these issues impact the results of TIME remains to be seen.

\section{Email and telephone interactions}

Individuals interested in taking part in the TIME study were able to contact study staff by a Freephone number, by email or by letter. To better understand the resource allocation required for a study using this methodology, we logged all telephone and email contacts for 1 month whilst recruitment was actively being pursued. During a 4-week period in June 2016, 3005 people registered with the study website. Of those, 1860 (62\%) went on to consent, complete enrolment and be randomised to a time of dosing. There were 1090 queries to the department about the study during this time: 457 (42\%) telephone calls, 522 (48\%) emails, and 108 (10\%) queries submitted using an online support tool on the study website. There 
were three additional queries where the method of contact was not recorded. The content of the queries varied: 116 (11\%) were IT problems, 199 (18\%) were clinically related questions, 701 (64\%) were administrative or general enquiries, $64(6 \%)$ were queries about withdrawing from the study, and 10 were complaints about study processes (Table 2).

This exercise was repeated in November 2016, when active recruitment had ceased. During this month, 61 individuals registered with the study website, 21 of whom went on to be randomised. There were 401 queries to the department: $300(75 \%)$ by email, $54(13 \%)$ by phone and $57(14 \%)$ via the online support tool. Of these, there were 8 (2\%) IT issues, $48(12 \%)$ clinical queries, 302 (75\%) administrative or general enquiries, $42(10 \%)$ queries on how to withdraw and 1 complaint (Table 3).

\section{Email and telephone interactions review}

The literature on email use in clinical studies is positive, with reports of it being used effectively in research [6-14]. The amount of resource that email and telephone enquiries required was large during the TIME recruitment phase. This is one area that should not be underestimated by researchers when planning study resources. The emails themselves became a key form of data. It became clear during the study that they potentially provide richly contextual prospective records, and this remains an area that is under-researched. Emails often recorded the context of volunteering and the motivations and priorities of volunteers. The volume of both emails and telephone calls highlighted the 'invisible work' of research staff during what are typically considered to be standard administrative tasks [49]. One area of concern highlighted by the literature was the lack of a standard process on how to store emails sent by participants containing confidential data. More attention should be paid to confidentiality, documentation and costing when using email communication in order to optimize its use [50].

\section{Discussion}

This work has several limitations. Primarily, the literature review was conducted exclusively using the PubMed library with generic search terms. Analysis of recruitment

Table 2 Participant contact methods and enquiries for June 2016

\begin{tabular}{lllll}
\hline & Telephone & Email & Study website & Other \\
\hline IT query & 65 & 43 & 6 & 2 \\
Clinical query & 48 & 100 & 51 & - \\
Administrative query & 332 & 321 & 47 & 1 \\
Withdrawal query & 9 & 54 & 1 & - \\
Complaint & 3 & 4 & 3 & - \\
\hline
\end{tabular}

IT Information technology
Table 3 Participant contact methods and enquiries for November 2016

\begin{tabular}{lllll}
\hline & Telephone & Email & Study website & Other \\
\hline IT query & 1 & 6 & 1 & - \\
Clinical query & 4 & 32 & 12 & - \\
Administrative query & 45 & 224 & 33 & - \\
Withdrawal query & 4 & 37 & 1 & - \\
Complaint & - & 1 & - & - \\
\hline
\end{tabular}

IT Information technology

strategies were limited to methods employed by the Medicines Monitoring Unit (MEMO), and authors were unable to analyse recruitment methods used by the supporting research networks.

Adherence to allocated treatment is under-reported in trial publications. This may be due to a concern about undermining study results. Researchers have attempted to improve participant adherence with varying success. However, loss to follow-up continues to be a problem. Lack of general participant understanding of research objectives and methodologies may contribute to this. There still appears to be confusion among both participants and researchers about when lack of adherence qualifies as withdrawal from the study or as loss to follow-up. Further work is necessary in clarifying study documentation to understand the differences and how to report these phenomena.

Further research is necessary regarding the now common use of email correspondence between researchers and participants. The proclivity to engage in email use in studies suggests that guidelines on best practices and efforts to secure patient confidentiality should be examined expeditiously.

\section{Conclusions}

The TIME study has successfully recruited more than 21,000 participants over a 2 -year period. The recruitment methods used have proven successful and costeffective in comparison to similar-sized studies. The total cost of this major trial is therefore an order of magnitude lower than the cost of a typical commercial clinic-based study. On the basis of experiences in the TIME study, as well as comparisons drawn from the literature, an online methodology and the use of EDC are viable for conducting clinical research. Numerous strategies should be incorporated to improve recruitment and optimise the process, and all available strategies should be used to improve study retention.

\footnotetext{
Acknowledgements

The authors acknowledge the contributions of project managers Wendy Saywood and Geraldine Mackle, project administrator Catriona Young, and the wider departmental staff of Medicines Monitoring Unit, University of Dundee, Dundee, UK. The authors also acknowledge the British Heart
} 
Foundation, the British and Irish Hypertension Society, the National Institute for Health Research (NIHR) Clinical Research Network, and the Scottish Primary Care Research Network.

\section{Funding}

The TIME study was funded by a grant from the British Heart Foundation, Greater London House, 180 Hampstead Road, London, NW1 7AW, UK.

\section{Availability of data and materials}

Anonymised data from the study can be made available to bona fide researchers on application.

\section{Authors' contributions}

DAR conceived of the idea for the study. DAR created the initial draft of the manuscript. TMM, RWVF, ISM and AR reviewed and edited the manuscript. All listed authors fulfil the requirements for authorship and agreed to submission of the manuscript in its current form. All authors read and approved the final manuscript.

\section{Ethics approval and consent to participate}

Ethical approval was obtained from the Tayside Committee on Medical Research Ethics (MREC reference 11/AL/0309; UKCRN identifier 17071). TIME is registered with the ISRCTN Registry (ISRCTN18157641). The trial was performed in line with good clinical practice guidelines and International Society of Pharmacoepidemiology (ISPE) Good Pharmacoepidemiology Practice Guidance. All participants consented to taking part in the TIME study.

\section{Consent for publication}

All listed authors fulfil the requirements for authorship and agree to submission of the manuscript in its current form.

\section{Competing interests}

The authors declare that they have no competing interests.

\section{Publisher's Note}

Springer Nature remains neutral with regard to jurisdictional claims in published maps and institutional affiliations.

Received: 16 June 2017 Accepted: 15 September 2017

\section{Published online: 23 November 2017}

\section{References}

1. de Melo-Martín I, Sondhi D, Crystal RG. When ethics constrains clinical research: trial design of control arms in "greater than minimal risk" pediatric trials. Hum Gene Ther. 2011;22(9):1121-7.

2. Rorie DA, Flynn RWV, Grieve K, Doney A, Mackenzie I, MacDonald TM, et al. Electronic case report forms and electronic data capture within clinical trials and pharmacoepidemiology. Br J Clin Pharmacol. 2017;83(9):1880-95.

3. Carvalho JC, Bottenberg P, Declerck D, van Nieuwenhuysen JP, Vanobbergen J, Nyssen M. Validity of an information and communication technology system for data capture in epidemiological studies. Caries Res. 2011;45(3):287-93.

4. Dillon DG, Pirie F, Rice S, Pomilla C, Sandhu MS, Motala AA, et al. Opensource electronic data capture system offered increased accuracy and costeffectiveness compared with paper methods in Africa. J Clin Epidemiol. 2014;67(12):1358-63.

5. Le Jeannic A, Quelen C, Alberti C, Durand-Zaleski I, CompaRec Investigators. Comparison of two data collection processes in clinical studies: electronic and paper case report forms. BMC Med Res Methodol. 2014;14:7.

6. Atherton H, Pappas $Y$, Heneghan C, Murray E. Experiences of using email for general practice consultations: a qualitative study. $\mathrm{Br} J$ Gen Pract. 2013; 63(616):e760-7.

7. Newhouse N, Lupiáñez-Villanueva F, Codagnone C, Atherton H. Patient use of email for health care communication purposes across 14 European countries: an analysis of users according to demographic and health-related factors. J Med Internet Res. 2015;17(3):e58.

8. Pattison N, O'Gara G, Rattray J. After critical care: Patient support after critical care. A mixed method longitudinal study using email interviews and questionnaires. Intensive Crit Care Nurs. 2015;31(4):213-22.
9. Cutrona SL, Roblin DW, Wagner JL, Gaglio B, Williams AE, Torres Stone R, et al. Adult willingness to use email and social media for peer-to-peer cancer screening communication: quantitative interview study. JMIR Res Protoc. 2013;2(2):e52.

10. Block G, Azar KM, Romanelli RJ, Block TJ, Hopkins D, Carpenter HA, et al. Diabetes prevention and weight loss with a fully automated behavioral intervention by email, web, and mobile phone: a randomized controlled trial among persons with prediabetes. J Med Internet Res. 2015;17(10):e240.

11. Cicolini G, Simonetti V, Comparcini D, Celiberti I, Di Nicola M, Capasso LM, et al. Efficacy of a nurse-led email reminder program for cardiovascular prevention risk reduction in hypertensive patients: a randomized controlled trial. Int J Nurs Stud. 2014;51(6):833-43.

12. Lancee J, van den Bout J, Sorbi MJ, van Straten A. Motivational support provided via email improves the effectiveness of internet-delivered selfhelp treatment for insomnia: a randomized trial. Behav Res Ther. 2013; 51(12):797-805

13. Torniainen-Holm M, Pankakoski M, Lehto T, Saarelma O, Mustonen $P$ Joutsenniemi $K$, et al. The effectiveness of email-based exercises in promoting psychological wellbeing and healthy lifestyle: a two-year followup study. BMC Psychol. 2016;4(1):21.

14. Tate DF, LaRose JG, Griffin LP, Erickson KE, Robichaud EF, Perdue L, et al. Recruitment of young adults into a randomized controlled trial of weight gain prevention: message development, methods, and cost. Trials. 2014;15(1):326.

15. Rorie DA, Rogers A, Mackenzie IS, Ford I, Webb DJ, Willams B, et al. Methods of a large prospective, randomised, open-label, blinded endpoint study comparing morning versus evening dosing in hypertensive patients: the Treatment In Morning versus Evening (TIME) study. BMJ Open. 2016;6(2):e010313.

16. Efficace F, Fayers P, Pusic A, Cemal Y, Yanagawa J, Jacobs M, et al. Quality of patient-reported outcome reporting across cancer randomized controlled trials according to the CONSORT patient-reported outcome extension: a pooled analysis of 557 trials. Cancer. 2015;121(18):3335-42.

17. Rorie DA, Rogers A, Mackenzie IS, Findlay E, MacDonald TM, et al. Treatment in the Morning versus Evening (TIME) study: feasibility of an online study. J Clin Trials. 2016:6:281.

18. Critchley JA, Capewell S. Mortality risk reduction associated with smoking cessation in patients with coronary heart disease: a systematic review. JAMA. 2003:290(1):86-97.

19. Biobank. About UK Biobank. http://www.ukbiobank.ac.uk/about-biobank-uk/. Accessed 27 Mar 2017.

20. NHS Research Scotland - Scottish Health Research Register. SHARE. http:// www.goshare.org.uk/. Accessed 27 Mar 2017.

21. Docmail. http://www.docmail.co.uk/. Accessed 27 Mar 2017.

22. Rorie D, Flynn R, McConnachie L, Mackenzie IS, Macdonald TM. Poster advertisements in practice waiting rooms to recruit patients to the Treatment In Morning vs. Evening (TIME) online study. Br J Clin Pharmacol. 2015;80(2):325-6.

23. Treweek S, Pitkethly M, Cook J, Kjeldstrøm M, Taskila T, Johansen M, et al. Strategies to improve recruitment to randomised controlled trials. Cochrane Database Syst Rev. 2010:4:MR000013.

24. Tompkins DA, Sides JA, Harrison JA, Strain EC. Recruitment techniques for alcohol pharmacotherapy clinical trials: a cost-benefit analysis. Addict Disord Their Treat. 2015;14(4):211-9.

25. Lara Jr PN, Paterniti DA, Chiechi C, Turrell C, Morain C, Horan N, et al. Evaluation of factors affecting awareness of and willingness to participate in cancer clinical trials. J Clin Oncol. 2005;23(36):9282-9.

26. Haynes R, Bowman L, Rahimi K, Armitage J. How the NHS research governance procedures could be modified to greatly strengthen clinical research. Clin Med (Lond). 2010;10(2):127-9.

27. Butt DA, Lock M, Harvey BJ. Effective and cost-effective clinical trial recruitment strategies for postmenopausal women in a community-based, primary care setting. Contemp Clin Trials. 2010;31(5):447-56.

28. Eisenstein EL, Collins R, Cracknell BS, Podesta O, Reid ED, Sandercock P, et al. Sensible approaches for reducing clinical trial costs. Clin Trials. 2008:5(1):75-84.

29. Christensen T, Riis AH, Hatch EE, Wise LA, Nielsen MG, Rothman KJ, et al. Costs and efficiency of online and offline recruitment methods: a web-based cohort study. J Med Internet Res. 2017;19(3):e58.

30. Batterham PJ. Recruitment of mental health survey participants using Internet advertising: content, characteristics and cost effectiveness. Int J Methods Psychiatr Res. 2014;23(2):184-91. 
31. Huynh L, Johns B, Liu SH, Vedula SS, Li T, Puhan MA. Cost-effectiveness of health research study participant recruitment strategies: a systematic review. Clin Trials. 2014;11(5):576-83.

32. Sinclair M, OToole J, Malawaraarachchi M, Leder K. Comparison of response rates and cost-effectiveness for a community-based survey: postal, internet and telephone modes with generic or personalised recruitment approaches. BMC Med Res Methodol. 2012;12(1):132.

33. Aung T, Haynes R, Barton J, Cox J, Murawska A, Murphy K, et al. Costeffective recruitment methods for a large randomised trial in people with diabetes: A Study of Cardiovascular Events iN Diabetes (ASCEND). Trials. 2016;17:286.

34. de Vries Herman J, Kloek Corelien JJ, de Bakker Dinny H, Dekker J, Bossen D, Veenhof $\mathrm{C}$. Determinants of adherence to the online component of a blended intervention for patients with hip and/or knee osteoarthritis: a mixed methods study embedded in the e-Exercise trial. Telemed e-Health. 2017. ahead of print. https://doi.org/10.1089/tmj.2016.0264.

35. Choudhry NK, Krumme AA, Ercole PM, Girdish C, Tong AY, Khan NF, et al. Effect of reminder devices on medication adherence: the REMIND randomized clinical trial. JAMA Intern Med. 2017;177(5):624-31.

36. Lynggaard V, Nielsen CV, Zwisler AD, Taylor RS, May O. The patient education - Learning and Coping Strategies - improves adherence in cardiac rehabilitation (LC-REHAB): a randomised controlled trial. Int J Cardiol. 2017;236:65-70.

37. de Jager RL, de Beus E, Beeftink MMA, Sanders MF, Vonken EJ, Voskuil M, et al. Impact of medication adherence on the effect of renal denervation: the SYMPATHY trial. Hypertension. 2017;69(4):678-84.

38. Caminati M, Senna G, Stefanizzi G, Bellamoli R, Longhi S, Chieco-Bianchi $F$, et al. Drop-out rate among patients treated with omalizumab for severe asthma: literature review and real-life experience. BMC Pulm Med. 2016;16(1):128.

39. Puts MTE, Monette J, Girre V, Wolfson C, Monette M, Batist G, et al. Participation of older newly-diagnosed cancer patients in an observational prospective pilot study: an example of recruitment and retention. BMC Cancer. 2009;9:277.

40. Peltola J, Peltola M, Auvinen A, Raitanen J, Fallah M, Keränen T. Retention rates of new antiepileptic drugs in localization-related epilepsy: a singlecenter study. Acta Neurol Scand. 2009;1 19(1):55-60.

41. Norvell DC, Dettori JR, Chapman JR. Enhancing clinical study retention rates to avoid follow-up bias: how do we keep our study participants from "the land of the lost"? Global Spine J. 2016;6(5):519-21.

42. Lindström D, Sundberg-Petersson I, Adami J, Tönnesen H. Disappointment and drop-out rate after being allocated to control group in a smoking cessation trial. Contemp Clin Trials. 2010;31(1):22-69.

43. Mohr DC, Goodkin DE, Masuoka L, Dick LP, Russo D, Eckhardt J, et al. Treatment adherence and patient retention in the first year of a phase-III clinical trial for the treatment of multiple sclerosis. Mult Scler J. 1999;5(3):192-7.

44. Somerson JS, Bartush KC, Shroff JB, Bhandari M, Zelle BA. Loss to follow-up in orthopaedic clinical trials: a systematic review. Int Orthop. 2016:40(11):2213-9.

45. Cleland JGF, Torp-Pedersen C, Coletta AP, Lammiman MJ. A method to reduce loss to follow-up in clinical trials: informed, withdrawal of consent. Eur J Heart Fail. 2004;6(1):1-2.

46. Kramer JR, Bayless ML, Lorenzi GM, Ziegler GK, Larkin ME, Lackaye ME, et al. Participant characteristics and study features associated with high retention rates in a longitudinal investigation of type 1 diabetes mellitus. Clin Trials. 2012;9(6):798-805

47. Mor M, Niv G, Niv Y. Patient retention in a clinical trial: a lesson from the rofecoxib (VIOXX) study. Dig Dis Sci. 2006;51(7):1175-8.

48. White L, Tanzi EL, Alster TS. Improving patient retention after botulinum toxin type A treatment. Dermatol Surg. 2006:32(2):212-5.

49. Townsend A, Amarsi Z, Backman CL, Cox SM, Li LC. Communications between volunteers and health researchers during recruitment and informed consent: qualitative content analysis of email interactions. J Med Internet Res. 2011;13(4):e84.

50. Dash J, Haller DM, Sommer J, Junod PN. Use of email, cell phone and text message between patients and primary-care physicians: crosssectional study in a French-speaking part of Switzerland. BMC Health Serv Res. 2016;16(1):549.

\section{Submit your next manuscript to BioMed Central and we will help you at every step:}

- We accept pre-submission inquiries

- Our selector tool helps you to find the most relevant journal

- We provide round the clock customer support

- Convenient online submission

- Thorough peer review

- Inclusion in PubMed and all major indexing services

- Maximum visibility for your research

Submit your manuscript at www.biomedcentral.com/submit

Biomed Central 